\title{
Etnoeducación: educación para la salud desde la diversidad cultural
}

Ethnoeducation: health education from cultural diversity

Etnoeducação: educação para a saúde a partir da diversidade cultural

\begin{abstract}
Alejandro Hernández-Martínez, Est. ${ }^{1}$ (D), Yuban Sebastián Cuartas-Agudelo, Est ${ }^{1}$ (D), Laura Herrera-Almanza, Est ${ }^{1}$ (D), Mabel Dahiana Roldan-Tabares, Est ${ }^{1}$ (D) Lina María Martínez-Sánchez, Bact., Esp, MSc. ${ }^{2}$ (D)

1. Estudiante de Medicina. Universidad Pontificia Bolivariana. Escuela de Ciencias de la Salud, Facultad de Medicina. Medellín, Colombia.

2. Bacterióloga, Especialista en Hematología, Magister en Educación Universidad Pontificia Bolivariana. Escuela de Ciencias de la Salud, Facultad de Medicina. Medellín, Colombia.
\end{abstract}

Correspondencia. Mabel Dahiana Roldan-Tabares. Universidad Pontificia Bolivariana, Calle 78 B N 72 a 109, Medellín, Colombia. Escuela de Ciencias de la Salud, Facultad de Medicina, teléfono: +57(4) 4488388. Email.mabel.roldan@upb.edu.co.

\section{INFORMACIÓN DEL ARTÍCULO:}

Artículo recibido: 26 de abril de 2020

Artículo aceptado: 08 de diciembre de 2020

DOI: https://doi.org/10.29375/01237047.3899

Cómo citar: Hernández-Martínez A, Cuartas-Agudelo YS, Herrera-Almanza L, Roldan-Tabares MD, MartínezSánchez LM. Etnoeducación: educación para la salud desde la diversidad cultural. MedUNAB. 2021; 24(1): 8091.Doi: https://doi.org/10.29375/01237047.3899

\section{RESUMEN}

Introducción. En un país multicultural como Colombia, la etnoeducación es un importante elemento en la educación médica, pues favorece la calidad de vida de minorías étnicas a partir de potencialidades comunitarias, prácticas, hábitos, experiencias y enfoques que promuevan el sentido global de la salud. El objetivo de este artículo fue realizar una revisión narrativa sobre los antecedentes de la etnoeducación y su impacto en la salud. El desarrollo de competencias interculturales en los estudiantes del área de la salud permitiría a los futuros profesionales desempeñar su rol con adecuada pertinencia cultural, respetando los valores, tradiciones e historia de las comunidades. Temas tratados: A nivel mundial, la 
etnoeducación ha sido un aspecto relevante, organizaciones internacionales han trabajado durante décadas para su implementación. A nivel nacional, se han creado diversos instrumentos normativos y reglamentación para la implementación de la etnoeducación, sin embargo, cerca del 86\% de las poblaciones étnicas no cuentan con acceso a educación que cumpla con los principios establecidos. La importancia de la etnoeducación se ha evidenciado en varios países, brindando resultados positivos como la disminución de la morbimortalidad con actividades educativas de promoción de la salud y prevención de la enfermedad. Para ello, es crucial que las acciones planteadas se integren con las percepciones culturales de las comunidades. Conclusión: Existen muchos lineamientos a nivel local, nacional e internacional, sin embargo, la etnoeducación continúa siendo un reto. Es necesario aumentar los esfuerzos para que la etnoeducación logre los objetivos que están planteados desde el punto de vista teórico.

Palabras claves:

Educación Médica; Diversidad Cultural; Promoción de la Salud; Participación de la Comunidad; Prestación de Atención de Salud; Salud de Poblaciones Indígenas.

\section{ABSTRACT}

Introduction. In a multicultural country such as Colombia, ethnoeducation is an important component of health education, because it promotes the quality of life of ethnic minorities based on the community's potential, practices, habits, experiences and approaches that promote overall health awareness. The purpose of this article is to perform a narrative review on the background of ethnoeducation and its impact on health. The development of inter-cultural skills among students in health fields would enable the future professional to perform their roles with adequate cultural relevancy, respecting the values, traditions and history of the communities. Topics discussed: Worldwide, ethnoeducation has been highly relevant, and some international organizations have worked on its implementation for decades. In Colombia, several legal and regulatory instruments have been developed to implement ethnoeducation. However, close to $86 \%$ of the ethnic populations do not have access to education in accordance with the established principles. The importance of ethnoeducation has been highlighted in several countries in that it has achieved positive results such as a reduction of morbidity and mortality through educational activities that promote health and help prevent diseases. To achieve this, it is essential that the planned activities be integrated into the communities' cultural perceptions. Conclusion: Although local, national and international guidelines have been established, ethnoeducation continues to be a challenge. It is necessary to increase efforts in order for ethnoeducation to achieve the objectives that have been set out from a theoretical perspective.

Keywords:

Medical Education; Cultural Diversity; Health Promotion; Community Participation; Delivery of Health Care; Health of Indigenous Populations.

\section{RESUMO}

Introdução. Em um país multicultural como a Colômbia, a etnoeducação é um elemento importante na educação médica, pois favorece a qualidade de vida das minorias étnicas a partir das potencialidades comunitárias, das práticas, dos hábitos, das experiências e abordagens que promovem o sentido global da saúde. O objetivo deste artigo foi realizar uma revisão narrativa sobre os antecedentes da etnoeducação e seu impacto na saúde. O desenvolvimento de competências interculturais nos alunos da área da saúde permitirá aos futuros profissionais desempenharem seu papel com adequada relevância cultural, respeitando os valores, tradições e história das comunidades. Tópicos discutidos: Em nível global, a etnoeducação tem sido um aspecto relevante e organizações internacionais trabalharam durante décadas para implementá-la. A nível nacional foram criados diversos instrumentos normativos e reguladores para a implementação da etnoeducação, no entanto, cerca de $86 \%$ das etnias não tem acesso a uma educação que cumpra os princípios estabelecidos. A importância da etnoeducação é evidente em diversos países, proporcionando resultados positivos como a redução da morbimortalidade com atividades educativas de promoção da saúde e prevenção de doenças. Para isso, é fundamental que as ações propostas estejam integradas às percepções culturais 
das comunidades. Conclusão: Existem muitas diretrizes a nível local, nacional e internacional, ainda assim, a etnoeducação continua a ser um desafio. É necessário aumentar os esforços para que a etnoeducação atinja os objetivos que são levantados a partir do ponto de vista teórico.

Palavras-chave:

Educação Médica; Diversidade Cultural; Promoção da Saúde; Participação da Comunidade; Assistência à Aaúde; Saúde de Populações Indígenas.

\section{Introducción}

La diversidad étnica y cultural ha creado una conciencia sobre la necesidad de gestionar por medio de políticas de participación de los grupos minoritarios étnicos y culturales recursos de la sociedad. El multiculturalismo es una respuesta sistemática e integral a la diversidad cultural y étnica, con carácter educativo, lingüístico, económico y social (1).

Según la Organización Mundial de la Salud (OMS), las diferencias en salud y bienestar son atribuibles a circunstancias específicas en las que "las personas nacen, crecen, viven, trabajan y envejecen", circunstancias que se conocen como los "determinantes sociales de salud" e incluyen leyes, políticas, economía, educación, condiciones de vida, etc. (2).

Los pueblos indígenas representan cómo las desigualdades sociales en conjunto con los determinantes sociales pueden afectar negativamente la salud. En 2009, la Organización de las Naciones Unidas (ONU) publicó un documento de análisis exhaustivo titulado "El estado de los pueblos indígenas del mundo", los resultados de este análisis ilustraron que el estado socioeconómico de las poblaciones indígenas en las naciones y territorios a nivel mundial es sustancialmente más bajo que el de otros que viven dentro de la misma zona. Los pueblos indígenas son menos educados que sus vecinos no indígenas y tienen menos oportunidades de medios de vida decentes disponibles para ellos (2).

La etnoeducación surge para sustentar los principios constitucionales que se refieren a la naturaleza multicultural de la nación colombiana y tiene sus inicios en $1976(3,4)$. Las experiencias en el campo de la educación indígena, y la educación afrocolombiana, encuentran en el marco de la política educativa un escenario para la puesta en práctica de la interculturalidad, como el principio fundamental de esta política que asume la posibilidad de educar de acuerdo con las culturas locales y en diálogo con la cultura global (3).

En un país multirracial y multicultural como Colombia, es extremadamente importante que los médicos tengan una buena comprensión de los elementos diversificadores como la cultura, las convicciones religiosas, los valores y las actitudes de sus pacientes, antes de que puedan proceder a tratar a sus pacientes con respeto y dignidad. La etnoeducación se convierte entonces en un importante elemento en la educación médica, tanto así que en una encuesta realizada a estudiantes de medicina, el 54\% consideró que la gestión de la diversidad es una habilidad imprescindible que se debe adquirir durante la formación médica (5). Incluso algunos expertos consideran que la educación médica debe centrarse en la mejor manera de integrar a los pacientes de minorías étnicas en el marco de atención médica existente (6).

La educación social para la salud se plantea como generadora de posibilidades que pueden mejorar y optimizar las condiciones de los sistemas de salud, la profesionalización de los agentes participantes en el campo socio-sanitario y la calidad de vida de las personas con el fin de lograr un desarrollo sostenible de las instituciones, los pueblos y las comunidades (7).

La promoción de la salud es un objetivo de la educación social, que busca la transmisión de la cultura para favorecer la calidad de vida, a partir de las potencialidades comunitarias, prácticas, hábitos, experiencias y enfoques que promuevan el sentido global de la salud (7).

Las políticas en salud pública apuestan por la mejora de los sistemas sanitarios mejorando la gestión del conocimiento, ciencia e innovación en salud, por medio de la participación institucional y la difusión de la salud con base en alianzas estratégicas con la academia, y redes de coordinación entre el sector privado y la sociedad civil (7).

La educación juega un papel primordial en el control cultural de los pueblos, aunque se ha intentado transformar el espacio académico, todavía hay mucho camino por recorrer (8). Es por lo anterior que el objetivo de este artículo fue realizar una revisión narrativa sobre los antecedentes nacionales e internacionales de la etnoeducación y su impacto en la salud, basados en una búsqueda de literatura $\mathrm{y}$ artículos de investigaciones originales en PubMed, Science Direct y Scielo durante noviembre de 2019 y marzo de 2020, con el uso de palabras clave y términos 
$\mathrm{MeSH}$ relacionados con educación médica, diversidad cultural, promoción de la salud, participación de la comunidad y prestación de atención de salud.

\section{Temas tratados}

\section{Educación y formación de recurso humano y competencias}

La mayoría de la literatura sobre etnoeducación en América Latina se centra en el análisis de marcos legales y potencial político, muy pocos han profundizado en los componentes pedagógicos y didácticos. En Colombia estos componentes permiten concluir, a partir de la observación de las comunidades indígenas y afrodescendientes en las que han sido implementados, que la educación ya no se entiende como una herramienta para destruir sus raíces e imponer estándares y valores de la cultura dominante, sino que por el contrario, la intención es redirigir la educación hacia los valores, las tradiciones y la historia cultural de las comunidades étnicas $(9,10)$.

Las competencias multidisciplinarias caracterizan la capacidad y la voluntad de los individuos que están en formación y les permiten aplicar sus conocimientos en sus actividades profesionales. Además del conocimiento, la competencia debe comprender la comunicación entre las diferentes disciplinas y la preparación psicológica para resolver los problemas de la actividad profesional y la disposición para obtener nuevos conocimientos en el proceso de estudiar otras disciplinas (11).

Para la buena ejecución del quehacer pedagógico es importante definir las competencias que deben adquirir los estudiantes, aspecto que constituye un desafío y determina el sentido de la enseñanza (12). Las competencias que adquieren los estudiantes deben estar de acuerdo con las exigencias sociales y culturales, de modo que permitan desarrollar el aprendizaje y adquirir nuevas habilidades y destrezas (12), posibilitando, de igual manera, la capacidad de articular e integrar tanto los conocimientos formales aprendidos en la institución académica, como los conocimientos personales que van de la mano con las experiencias de vida (13). El desarrollo de competencias interculturales en los estudiantes del área de la salud permitiría a los futuros profesionales de la atención primaria desempeñar su rol con adecuada pertinencia cultural, asumiendo las características que impone la época actual tanto para la relación con los usuarios, como para su comprensión, en tanto seres holísticos y culturalmente diversos (13).
De acuerdo con el modelo de competencias comunicativas interculturales de Byram (14), se plantea la generación de saberes adquiridos en vez de aprendizaje, lo que permite el contacto de diferentes culturas, incluyendo la propia. Las competencias interculturales son: actitudes en el saber ser que permiten reconocer lo que se tiene para dar paso a la curiosidad y reconfigurar la subjetividad; y saberes para la comprensión de la información relativa a otras culturas, tanto en lo interno como en lo externo (4).

Según Villegas y Lucas (15), es necesario definir las competencias docentes que son requeridas en un entorno multicultural que permitan lograr completamente la transformación de la sociedad, que es el objetivo final de la educación multicultural. Los maestros deben tener seis rasgos principales: conciencia sociocultural; actitud de reconocer adecuadamente a los estudiantes con diferentes antecedentes; responsabilidad y habilidad para actuar como un agente de cambio, para hacer escuelas y una sociedad más justa; identificar a los estudiantes de cerca, y un estilo de enseñanza adecuado. Acar y Gürol (1) definieron tres dimensiones para identificar las competencias multiculturales de los docentes, que son:

- Primera dimensión: componentes de competencia cultural (conciencia, conocimiento, actitud, habilidades).

- Segunda dimensión: contextos de competencia cultural (personal, profesional, institucional y social).

- Tercera dimensión: focos de competencia cultural (perspectivas socioculturales, estudiante, enseñanza y transformación).

El docente debe tener un comportamiento proactivo, teniendo como base una mayor sensibilidad a las necesidades de formación, determinadas por los aspectos socioeconómicos asociados para maximizar el potencial de los recursos humanos, en el contexto de la apertura al aprendizaje permanente (16). Los docentes como guías en situaciones o experiencias de práctica clínica, deben establecer espacios de reflexión sobre dichas experiencias para fomentar la reflexión individual y colectiva, incluyendo actividades destinadas a que los estudiantes fortalezcan sus competencias interculturales. De esta manera, se da lugar a la creación e implementación de actividades de promoción y prevención con participación activa de los estudiantes y la población, y la incorporación del trabajo interdisciplinario en el desarrollo de las experiencias clínicas (13). 


\section{Antecedentes internacionales, nacionales y locales}

A nivel mundial, la etnoeducación ha sido un aspecto relevante evidenciado en diferentes instrumentos normativos de orden internacional. En la Declaración Universal de Derechos humanos de 1948, se establece que la educación posibilita el pleno desarrollo del individuo y a la promoción de la paz. En el convenio de la OIT No. 169, se aborda el problema de la educación en pueblos indígenas, y allí se expresa la importancia de la adecuación de los programas educativos según la necesidad de la población, con el objetivo de brindar conocimientos generales que les permitan a estas comunidades participar plenamente y en igualdad de condiciones en su grupo étnico y su país (17).

Adicionalmente, entidades a nivel mundial como la Organización de las Naciones Unidas para la Educación, la Ciencia y la Cultura (UNESCO), cuentan con más de 20 años trabajando para introducir e implementar la etnoeducación como aspecto clave de la educación para la paz, brindando diversas directrices en las cuales se enseña acerca de las lenguas, historias y culturas de las minorías, debido a que la diversidad cultural constituye un gran tesoro para la humanidad, y esta solo puede ser promovida en la medida en que se garantiza el cumplimiento de sus derechos humanos (14). A su vez, la UNESCO estableció tres principios de la etnoeducación (17):

- "Respeta la identidad cultural del educando impartiendo a todos una educación de calidad que se adecúe y adapte a su cultura."

- "Enseña a cada educando los conocimientos, las actitudes y las competencias culturales necesarias para que pueda participar plena y activamente en sociedad."

- "Enseña a todos los educandos los conocimientos, actitudes y las competencias culturales que les permiten contribuir al respeto, el entendimiento y la solidaridad entre individuos, entre grupos étnicos, sociales, culturales y religiosos y entre naciones."

En Colombia, un país con gran diversidad cultural, la etnoeducación constituye una necesidad y un gran reto, por ello, a lo largo de la historia se han creado instrumentos normativos relacionados a favor y en contra de la misma (18) (Figura 1).

Figura 1. Línea del tiempo con algunos Instrumentos Normativos de Colombia

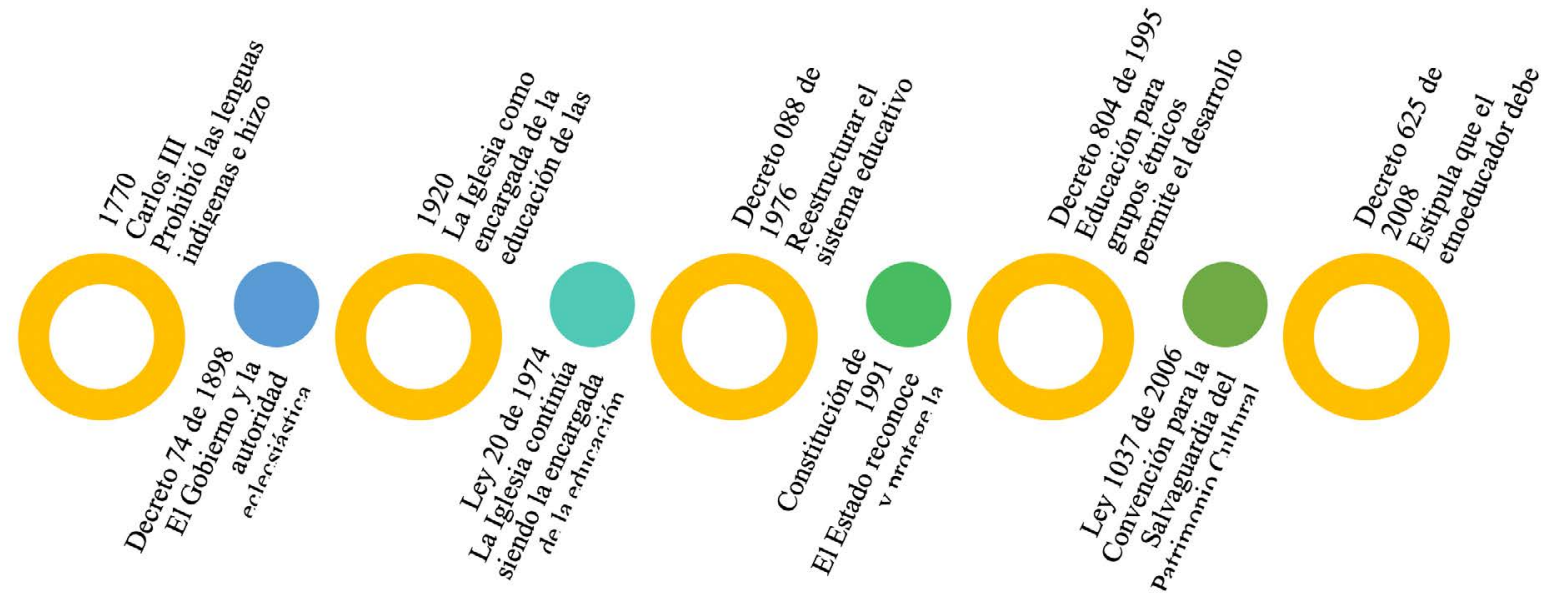

Lesión glútea altamente vascularizada con múltiples vasos aferentes de gran tamaño sin opacificación venosa temprana.

Fuente: Arbeláez Jiménez J, Vélez Posada P. La etnoeducación en Colombia: una mirada indígena (18)

Con la finalidad de favorecer el abordaje en salud de grupos étnicos, en los cuales la atención se realice siempre respetando y conociendo la cultura de los mismos, en el país se ha optado por la educación del personal médico. Uno de los cursos disponibles, totalmente gratuito, se encuentra en la plataforma de SOFIA PLUS, el portal de oferta educativa del Servicio Nacional de Aprendizaje (SENA), denominado "Atención en salud con pertinencia étnica", en el cual, en 40 horas, se educa sobre la normatividad, el sistema médico tradicional de los grupos étnicos y su relación con la medicina occidental, la comunicación y concertación con los grupos étnicos, los procesos de atención en salud, entre otros (19).

Así mismo, debido a que el lenguaje puede representar una barrera en la educación en salud para los grupos 
étnicos, el grupo Piraguas, un grupo de proyección social de la Universidad Pontificia Bolivariana de Medellín, decidió publicar un libro que se denomina Formación en hábitos saludables bajo el modelo de prevención de enfermedades, escrito en el idioma español y la lengua emberá, para facilitar la comprensión por parte de grupos étnicos. Adicionalmente, cuenta con ilustraciones que representan a los grupos indígenas, todo ello con la finalidad de formar en hábitos saludables que disminuyen la frecuencia de enfermedades infectocontagiosas frecuentes (20).

Lamentablemente, a pesar de toda la reglamentación para la implementación de la etnoeducación en el país, cerca del $86 \%$ de las poblaciones étnicas no cuentan con acceso a educación que cumpla con los principios establecidos por la UNESCO expuestos previamente, por ello es de vital importancia continuar con el desarrollo de estrategias que permitan la ejecución de procesos de enseñanza etnoeducativos, y que esto se constituya en una verdadera política pública basada en el respeto por la diversidad étnica (21-23).

En cuanto a la normativa colombiana que rige la etnoeducación, la Constitución Política de Colombia de 1991, en su artículo 7, reconoce y protege la diversidad étnica y cultural de la Nación; por otra parte, el Ministerio de Educación Nacional es el encargado de velar por el apoyo a la educación de grupos étnicos, quienes, a su vez, según el capítulo 3 de la Ley 115 de 1994, pueden plantear modelos educativos que estén acordes a su estilo de vida e intereses, con la finalidad de disminuir la inequidad o discriminación de estas comunidades (24). Además, según el decreto 1122 de 1998, se deberán impartir saberes, prácticas, valores y conocimientos que ayuden a promover la interculturalidad e integración de las comunidades vulnerables en todas las instituciones educativas (25).

Las líneas para apoyar estos procesos actúan según varios mecanismos, entre los que destacan el mantenimiento de la lengua materna como fundamento principal, la facilitación de formación de educadores específicos en este ámbito, asesorías personalizadas para el desarrollo educativo, la regulación de la intervención de organismos internacionales en el proceso etnoeducativo y la actualización e investigación para etnoeducadores (24).

El departamento del Huila, en su plan de desarrollo "El camino es la educación" para el período 2016-2019, se comprometió con la adquisición de competencias y participación en educación de las diferentes poblaciones, apostando por el mejoramiento de la etnoeducación para que todas las personas cuenten con acceso y permanezcan exitosamente en el sistema educativo (26).

Por otro lado, en Ipiales, Nariño, mediante el decreto 176 de 2016, se crea la Mesa Técnica de Concertación para el Fortalecimiento de la Política Educativa de los Pueblos Indígenas, allí se construyen acuerdos y acciones para el beneficio de las comunidades indígenas en materia etnoeducativa (27).

En el departamento de La Guajira, el plan de desarrollo "Un nuevo tiempo: 2017-2019", describe la etnoeducación como una adquisición permanente de conocimiento, disciplina y creatividad, todo esto mediante una alfabetización digital y lingüística, pero sin dejar de lado (como lo plantea la Ley 115 de 1994) el wayuunaiki, su lengua materna (28).

En Medellín, existen instrumentos normativos a nivel local con el objetivo de introducir la etnoeducación al proceso de enseñanza en diversos niveles, entre ellos encontramos el Acuerdo 085 de 2018, en el cual se institucionaliza la etnoeducación para la ciudad, al igual que en el Acuerdo No 128 de 2018 (29,30). A su vez, en el Plan de Desarrollo anterior de la ciudad, es decir, 20162019, se ejecutó el Programa Diversidad Étnica, con el propósito de promover la inclusión social de los grupos étnicos siempre promoviendo la equidad y respeto por la cultura (31).

\section{Importancia de la educación para todos}

La inclusión en la educación continúa siendo un concepto abstracto, pues, dependiendo del contexto geográfico y cultural, tiene como objetivo integrar al estudiante con discapacidad al sistema educativo o adaptar el sistema educativo a la diversidad de los estudiantes. Lo que sí está claro es que debe concebirse como una búsqueda constante de solución a la diversidad, minimización y eliminación de las barreras de cualquier tipo y ha de propender por los buenos resultados escolares (32).

La educación que tiene como objetivo respetar y considerar la diversidad cultural es un reto para todos, especialmente para los profesores, ya que se requiere un énfasis especial en las interacciones interpersonales y socioculturales. Para cumplir con esto, se han planteado diferentes estrategias tales como diálogos, similitudes o diferencias entre conocimientos de las personas para crear conexiones entre estas (33).

Según Valcarce Fernández, las escuelas que son o pretenden ser inclusivas deben cumplir con cinco requisitos: 
flexibilidad, informalidad, horizontalidad, además de ser participativas y competentes. Estas características tienen como principal objetivo abarcar la diversidad estudiantil para posteriormente ampliar los resultados al exterior de la escuela, es decir, a la comunidad y posteriormente a la sociedad (34).

La importancia de la educación para toda la población se ha evidenciado en países como México, donde se han mejorado los resultados escolares de los niños que participan en programas de educación intercultural. Sumado a lo anterior, Perú, Ecuador y Bolivia, han logrado crecer en materia de derechos y beneficios sociales a través del ámbito educativo, demostrando así la preponderancia de la educación (35).

La etnoeducación se ha implementado en varios países, Colombia entre ellos, pues las poblaciones indígenas han adelantado proyectos que radican en la reinvindicación y defensa de la educación propia. Los indígenas reconocen la importancia y necesidad de educarse, por eso, la creación de planes de estudio flexibles y adaptados a las diferentes poblaciones étnicas es el resultado de las movilizaciones de estos grupos de personas (36). Para lograr una educación inclusiva se debe identificar el contexto, la experiencia y la necesidad local, además es indispensable el hecho de poseer un acceso a educación de calidad en igualdad de oportunidades para todos. Esto debe orientar a la totalidad de los participantes, tanto docentes como estudiantes, a convertir sus prácticas en evidencias de trabajo en equipo, pero también la orientación personalizada para alcanzar resultados positivos (37).

Una educación inclusiva, permite el desarrollo educativo adecuado de personas pertenecientes a diferentes grupos poblacionales tales como discapacitados, comunidades indígenas, afrodescendientes, embarazadas y desmovilizados de grupos al margen de la ley, entre otros $(37,38)$. Además, la etnoeducación actualmente es una puerta abierta para inmiscuir a todas las personas en nuevas experiencias y humanizar las relaciones sociales, potenciando individualmente las personas y por ende, la sociedad (39).

\section{Etnoeducación, promoción de la salud y prevención de la enfermedad}

Desde 1986, la Conferencia Internacional de Ottawa definió la promoción de la salud como el proceso de capacitar a las personas y a las comunidades para que aumenten el control sobre los determinantes de la salud, y por lo tanto, mejoren su salud (40). Así mismo, la promoción de la salud también se ha definido como el proceso de educar a las personas para que aumenten el control sobre su salud y esta mejore notablemente (41, 42). Por su parte, la prevención se refiere a la realización de un análisis completo del individuo y el contexto en el que este se desarrolla, con el fin de reconocer aquellos factores de riesgo que posee y aquellos que pueden llegar a desarrollar en un futuro y que de cierta manera favorezcan la aparición de condiciones que afecten su salud (43).

La Organización Panamericana de la Salud (OPS) plantea tres mecanismos a través de los cuales se puede fomentar la promoción de la salud $(42,44)$ :

- El autocuidado, es decir, las acciones que la persona realiza por su propia cuenta para beneficio de su salud.

- La ayuda mutua o comunitaria que las personas realizan para ayudarse unas a otras de acuerdo con el contexto en el que se desenvuelven.

- La creación de entornos sanos que favorezcan la salud por medio de la educación, como por ejemplo la creación de escuelas saludables que se convierten en escenarios potenciadores para la capacitación de la población, en las que la educación para la salud y la promoción de la salud son opciones pedagógicas para el desarrollo humano.

Actualmente la promoción de salud se ha convertido en un práctica que implica aspectos como la educación, formación, investigación, legislación, coordinación de políticas y desarrollo comunitario, por lo que hoy en día se han reportado múltiples experiencias que integran a las comunidades indígenas en los procesos de promoción y prevención, como es el caso de los jóvenes Inuit en el territorio de Nunavut (Canadá), quienes se apropiaron de un proyecto de prevención del suicidio en su comunidad, así como la nación Navajo en los Estados Unidos, con jardines comunitarios para la promoción y prevención de la diabetes y la obesidad a través de los buenos hábitos alimenticios (45-47). El impacto de la experiencia de los jardines comunitarios en la nación Navajo se encuentra en evaluación por parte de los autores; sin embargo, este tipo de intervenciones de educación en salud han mostrado resultados positivos en cuanto al consumo de frutas y verduras en otras poblaciones (47). Es crucial que este tipo de intervenciones y procesos se integren a las percepciones culturales de las comunidades, debido al impacto de estas percepciones en el comportamiento de los individuos y en la interacción con la intervención (48). 
En relación con temas dedicados a la reducción de la mortalidad y la prevención del suicidio, Harlow et al., identificaron nueve programas de educación dirigidos a jóvenes indígenas de Estados Unidos y Australia. Estos programas se caracterizaron por su adaptación a la cultura indígena y por la utilización de estrategias de educación sobre suicidio, entrenamiento a líderes y a la comunidad (49). Esta revisión sistemática reporta como resultado de dichos programas menor ideación y gestos suicidas tras la intervención; no obstante, se requieren estudios con mediciones más controladas, pues son pocos los estudios con metodología cuantitativa aleatorizada que controle los desenlaces (49).

En una revisión de Araujo et al., se encontraron 22 estudios realizados en Estados Unidos y Canadá sobre diversos tipos de intervenciones en salud en poblaciones indígenas, con evidencia de moderada o alta calidad que reportó efectividad de estrategias en educación médica para prevenir la depresión, las caries en la niñez y el uso de asientos de seguridad para los niños (50).

De esta manera, se ha enfatizado en la necesidad de aproximaciones holísticas, comunitarias y centradas en la familia y en los jóvenes al momento de diseñar programas de promoción y prevención para lograr tener efecto en las comunidades (51). Esto puede lograrse a través de la estrategia de investigación participativa basada en la comunidad (CBPR, por sus siglas en inglés), la cual ha demostrado fortalecer la capacidad investigativa y formar vínculos entre los investigadores y las comunidades (52). Las estrategias CBPR buscan fomentar el compromiso y la participación de las comunidades a través de las dinámicas colaborativas con los investigadores teniendo en cuenta la evidencia disponible para las intervenciones (52).

\section{Educación y morbimortalidad}

La educación se ha convertido en una herramienta para lograr la salud, sobre todo cuando se desarrolla a través de la participación de las personas, convirtiéndose incluso en promoción de la salud (45). La participación de las comunidades étnicas debe estar motivada por un mejoramiento continuo de la calidad de la salud y una reducción de la morbimortalidad (45).

La educación tiene un efecto directo en algunos determinantes de la mortalidad; la mortalidad del niño, por ejemplo, está influida por las creencias y valores que la madre tenga sobre el cuidado de su hijo y el comportamiento ante sus enfermedades (53). Según estadísticas de la OPS, las madres con menor grado de escolaridad y sus hijos tienen resultados de salud menos favorables que las madres con mayor nivel de educación. La tasa de mortalidad infantil entre bebés menores de un año cuyas madres tienen bajo grado de educación y los hijos de aquellas que tienen educación secundaria o superior, llega a ser siete veces mayor en El Salvador, tres veces mayor en Bolivia, Guatemala, Colombia y República Dominicana, y el doble en Perú (54).

Refiriéndose específicamente a las comunidades indígenas, un estudio sobre intervenciones en los estilos de vida en pueblos nativos americanos en los Estados Unidos concluyó que una reducción de peso moderada a leve se asoció a una reducción sustancial del riesgo a largo plazo de diabetes, reportando una reducción del $64 \%$ (IC 95\% 54 a 72) en aquellos con una pérdida $>5 \%$ comparados con quienes perdieron $<3 \%$ del peso corporal (55). Por otro lado, también se ha visto un impacto de este tipo de enfoques educativos en la morbimortalidad por trauma en estos pueblos: se ha logrado disminuir las heridas por accidentes asociados a vehículos de motor en estas poblaciones desde estrategias educativas basadas en evidencia aplicadas a las comunidades (56).

La diabetes sigue siendo una enfermedad que trae consigo una alta carga de morbimortalidad y las poblaciones indígenas no están exentas de esto. Bajo esta premisa, en Estados Unidos un servicio de salud indígena planteó un programa que incluyó la entrega de insumos para monitoreo de glucemia, referencia a especialistas y educación continua a los indígenas con esta patología a través de equipos multidisciplinarios de apoyo, logrando disminución de una unidad en los niveles de hemoglobina glicosilada y la reducción de aproximadamente $60 \%$ en la incidencia de amputaciones $(50,57)$.

Así mismo, otro estudio mostró un impacto en la mejoría de la calidad de la dieta en adolescentes de una comunidad de nativos de Alaska al realizar intervenciones educativas en los colegios de dicha comunidad, al mismo tiempo que permitía a estos adolescentes conectarse con las tradiciones culinarias de su grupo humano (58). Otro ejemplo podemos encontrarlo en México, donde un programa educativo en salud sexual y reproductiva aplicado a mujeres indígenas y adolescentes marginadas mostró que ambos grupos aumentaron de forma significativa su conocimiento a corto plazo, así como las conductas y actitudes respecto a la salud reproductiva y el uso del condón, mostrando la efectividad de la implementación de programas educativos orientados a ciertos grupos poblacionales $(59,60)$.

Actualmente, las estrategias de etnoeducación siguen siendo herramientas de primera mano para la reducción de 
la mortalidad en comunidades indígenas. Desde el 2015, la OPS/OMS, el Fondo de Población de las Naciones Unidas (UNFPA), el Fondo de las Naciones Unidas para la Infancia (UNICEF), y el Programa Mundial de Alimentos (PMA), se unieron para desarrollar un plan de acción que tiene como objetivo contribuir con la reducción de la mortalidad materna y neonatal en comunidades indígenas en Colombia, desarrollando talleres asociados con la atención del embarazo, parto, posparto y atención del recién nacido (61).

\section{Conclusiones}

Aunque existen muchos lineamientos a nivel local, nacional e internacional que respaldan la etnoeducación, esta continúa siendo un reto para los diferentes actores del sector educativo, social y sanitario, ya que aunque se ha intentado transformar el espacio académico todavía hay mucho camino por recorrer. Ello se debe a que se requiere de una muy buena comunicación entre las diferentes disciplinas y la preparación psicológica para resolver los problemas de la actividad profesional y la disposición para obtener nuevos conocimientos en el proceso de estudiar otras disciplinas. Sin embargo, en la educación y formación de recurso humano, las competencias interculturales en los estudiantes del área de la salud no hacen parte del plan de estudios obligatorio. Debido al gran impacto positivo que se puede alcanzar con la etnoeducación en salud, es necesario aumentar los esfuerzos para que esta logre los objetivos que están planteados desde el punto de vista teórico, ya que en la práctica aún siguen siendo vagos.

\section{Conflicto de interés}

No se declara ningún conflicto.

\section{Fuentes de financiación}

Por tratarse de una revisión de tema, no fue necesario ningún tipo de financiación para llevarse a cabo.

\section{Referencias}

1. Acar Y, Gürol M. Conceptual Framework Regarding the Multicultural Education Competencies of Teachers. H. U. Journal of Education. 2015;30(1):0114.

2. Reap M, Pornwattanavate S, Thame C, Van der Putten M. The social determinants of health of the
Urak Lawoi' of southern Thailand. BMC Public Health. 2020;20(1):197. https://doi.org/10.1186/s12889-020-8283-y

3. Castillo E, Hernández E, Rojas A. Los etnoeducadores: esos nuevos sujetos de la educación colombiana. Rev. colomb. educ. 2005;(48):38-54. https://doi. org/10.17227/01203916.7716

4. Izquierdo M. Educación en contextos multiculturales: experiencia etnoeducativa e intercultural con población indígena del Resguardo Embera Chamí - Mistrató, Risaralda - Colombia. Zona Próxima. 2018;29:1-22. http://dx.doi.org/10.14482/zp.29.0002

5. Siraj HH, Zamzam R, Ismail J, Mohamad N. Managing Diversity: A 'Must-Have' Skill for Medical Students. Procedia Soc Behav Sci. 2011;18: 379-38. https://doi.org/10.1016/j.sbspro.2011.05.054

6. Ali MA, Halim MU. Recent ideas on ethnic diversity in medical education: one step forward, two steps back? Medical Education. 2014 Apr;48(4):451. https://doi.org/10.1111/medu.12383

7. del Pozo F. Educación social para la salud: proyección, acción y profesionalización. Rev. Méd. Risaralda. 2013;19(1):75-80. http://dx.doi. org/10.22517/25395203.7881.

8. Triviño L, Palechor L. Logros y retos de la etnoeducación en Colombia. Universitas. Revista de Ciencias Sociales y Humanas. 2006;(7):145-181. https://doi.org/10.17163/uni.n7.2006.06

9. García W, Martín, M. Revisión histórica del fenómeno educativo indígena en el nororiente del Cauca (Colombia). Revista Española de Educación Comparada. 2011;18:279-302.

10. Flores I, Palacios N. Cultural and Intercultural Education: Experiences of Ethnoeducational Teachers in Colombia. Australian Journal of Teacher Education. 2018;43(7):62-81. https://doi.org/10.14221/ ajte.2018v43n7.4

11. Kachalov N, Kornienko A, Kvesko R, Kornienko A, Kvesko S, Chaplinskaya Y. Interdisciplinary Competences and Their Status Role in the System of Higher Professional Education. Procedia - Social and Behavioral Sciences. 2015; 206:429-33. https://doi. org/10.1016/j.sbspro.2015.10.078

12. Roys R.Competenciasdocentes:Desdeunaperspectiva Etnoeducativa y Tecnológica. Praxis. 2016;12(1):7889. https://doi.org/10.21676/23897856.1849

13. Veliz-Rojas L, Bianchetti-Saavedra AF, SilvaFernández M. Competencias interculturales en la atención primaria de salud: un desafío para la educación superior frente a contextos de diversidad cultural. Cad Saúde Pública. 2019:35(1):e00120818. https://doi.org/10.1590/0102-311x00120818

14. Byram M. Teaching and Assessing Intercultural Communicative Competence. Clevedon: Multilingual Matters ltd; 1997. 
15. Villegas A, Lucas T. Preparing Culturally Responsive Teachers: Rethinking the Curriculum. Journal of Teacher Education. 2002;53(1):20. https://doi. org/10.1177/0022487102053001003

16. Serdenciuc N. Competency-Based Education Implications on Teachers' Training. Procedia - Social and Behavioral Sciences. 2013;76:754-58. https://doi. org/10.1016/j.sbspro.2013.04.200

17. UNESCO. Directrices de la UNESCO sobre la educación intercultural [Internet]. Paris: UNESCO; 2006[citado 21 de abril de 2020]. Recuperado a partir de https://unesdoc.unesco.org/in/documentViewer. xhtml? $\mathrm{v}=2.1 .196 \& \mathrm{id}=\mathrm{p}:$ :usmarcdef 0000147878 spa\&file =/in/rest/annotation S V C/ Download WatermarkedAttachment/ attach import 507b74f8-9a4a-41a9-b914cce 1 db014c7b\%3F \%3 D 147878spa. pdf\&locale $=$ es \&multi $=$ true $\&$ ark $=/$ ark $: / 48223 /$ pf0000147878 spa/PDF/147878spa. pdf\# $\% 5 \mathrm{~B} \% 7 \mathrm{~B} \% 22$ num $\% 22 \% 3 \mathrm{~A} 73 \% 2 \mathrm{C} \% 22 \mathrm{gen} \%$ $22 \% 3 \mathrm{~A} 0 \% 7 \mathrm{D} \% 2 \mathrm{C} \% 7 \mathrm{~B} \% 22$ name $\% 22 \% 3 \mathrm{~A} \% 22 \mathrm{X}$ YZ \%22\%7D\%2C28\%2C623\%2Cnull\%5D

18. Arbeláez Jiménez J, Vélez Posada P. La etnoeducación en Colombia: una mirada indígena [Internet]. Medellín: EAFIT; 2008 [citado 21 de abril de 2020]. Recuperado a partir de https://repository.eafit.edu.co/ handle/10784/433

19. Servicio Nacional de Aprendizaje. Atención en Salud con Pertinencia Étnica. Bogotá: SENA; 2018.

20. Hernández Sarmiento JM, Álvarez Hernández LF, Restrepo Arango M, Vargas Grisales N, Martínez Sánchez LM, Roldán Tabares MD, et al. Formación en hábitos saludables bajo el modelo de prevención de enfermedades [Internet]. Medellín: Universidad Pontificia Bolivariana; 2018 [citado 7 de julio de 2020]. Recuperado a partir de https://repository.upb. edu.co/handle/20.500.11912/3847

21. Save the Children. Etnoeducación, un reto por el respeto y reconocimiento de nuestra población [Internet]. Bogotá: Save the Children. 2016 [citado 21 de abril de 2020]. Recuperado a partir de https:// www.savethechildren.org.co/articulo/etnoeducaciónun-reto-por-el-respeto-y-reconocimiento-de-nuestrapoblación

22. Enciso Patiño P. Estado del arte de la etnoeducación en Colombia con énfasis en política pública [Internet]. Bogotá: Ministerio de Educación Nacional; 2004 [citado 21 de abril de 2020]. Recuperado a partir de http://red-ler.org/estado-arte-etnoeducacioncolombia.pdf

23. Romero Medina A. Educación por y para indígenas y afrocolombianos: las tecnologías de la etnoeducación [Internet]. Bogotá: Universidad Javeriana; 2010 [citado 21 de abril de 2020]. Recuperado a partir de
https://revistas.javeriana.edu.co/index.php/MAGIS/ article/view/3534

24. Congreso de la República de Colombia. [8 febrero 1994]. Ley 115 de 1994. [Citado 07 Jul 2020]. Recuperado a partir de https://www.funcionpublica. gov.co/eva/gestornormativo/norma pdf.php?i=292.

25. Presidencia de la República de Colombia. [8 mayo 1995]. Decreto 804 de 1995. [citado 07 Jul 2020]. Recuperado a partir de https://www.mineducacion. gov.co/1759/w3-article-103494.html? noredirect=1.

26. Gobernación del Huila. Plan de desarrollo 2016-2019. "El camino es la educación". 2016. Colombia. [citado el 7 Jul 2020]. Recuperado a partir de https:/www. huila.gov.co/documentos/526/plan-de-desarrollo/

27. Alcaldía Municipal de Ipiales. Decreto 176 de agosto 16 de 2016. Colombia [citado el 7 Jul 2020]. Recuperado a partir de https://ipialesnarino. micolombiadigital.gov.co/sites/ipialesnarino/content/ files/000063/3119 decreto-176-de-2016ago-16.pdf

28. Gobernación de La Guajira. Plan de desarrollo por la Guajira 2017-2019 “Un nuevo Tiempo". 2017. Colombia. [citado el 7 Jul 2020]. Recuperado a partir de http://www.laguajira.gov.co/web/attachments/ article/4221/Plan\%20de \%20Desarrollo\%2020172019.pdf.

29. Acuerdo 085 de 2018 [Internet]. Medellín: Gaceta Oficial (9 de agosto de 2018) [citado 21 de abril de 2020]. Recuperado a partir de https://www.medellin. gov.co/normograma/docs/a conmed 00852018. $\underline{\mathrm{htm}}$

30. Medellín. Concejo. Secretaria General. Concepto 28 [Internet]. Medellín: Concejo de Medellín; 2018 [citado 21 de abril de 2020]. Recuperado a partir de https://www.medellin.gov.co/normograma/docs/cto sgmed 0000028 2018.htm

31. Medellín. Alcaldía. Plan de desarrollo 2016-2019 [Internet]. Medellín: Alcaldía de Medellín; 2016 [citado 21 de abril de 2020]. Disponible en: https:// www.medellin.gov.co/irj/go/ $/ \mathrm{km} /$ docs $/$ pccdesign/ SubportaldelCiudadano 2/PlandeDesarrollo 0 17/ Publicaciones/Shared \%20 Content/ Documentos/2016/Proyecto $\% 20 \mathrm{de} \% 20$ Acuerdo $\% 20$ Plan\%20de\%20Desarrollo.pdf

32. Parra C. Educación inclusiva: un modelo de diversidad humana. Revista Educación y Desarrollo Social. 2011;5(1):139-150.

33. Santos-Baptista GC. Tables of contextual cognition: a proposal for intercultural research in science education. Cult Stud of Sci Educ. 2017;13:845-63. https://doi.org/10.1007/s11422-017-9807-3

34. Valcarce M. De la escuela integradora a la escuela inclusiva. Innovación Educativa. 2011;21:119-131.

35. Nadenka BM. Teaching Based on Traditional Knowledge of Wayuu Ethnic Communities. 
Educación y Educadores. 2019;22(2):237-55. https:// doi.org/10.5294/edu.2019.22.2.4

36. Ferrero E. Ethno-Education (Etnoeducación) in La Guajira, Colombia: Shaping Indigenous Subjectivies Within Modernity, Neoliberal Multiculturalism and the Indigenous Struggle. Latin American and Caribbean Ethnic Studies. 2015;10(3):288-314. https://doi.org/10.1080/17442222.2015.1059542

37. Acuña LM, Cárdenas ML. Inclusive Education and ELT Policies in Colombia: Views From Some PROFILE Journal Authors. Profile. 2017;19(1): 12136. https://doi.org/10.15446/profile.v19n1.61075

38. Echeita G, Ainscow M. La educación inclusiva como derecho: marco de referencia y pautas de acción para el desarrollo de una revolución pendiente. Revista de Didáctica de la Lengua y la Literatura. 2011;12:2646.

39. Meneses YA. La etnoeducación afrocolombiana: conceptos, trabas, patriarcado y sexismo. A propósito de los 20 años de la Ley General de educación 115 de 1994. Rev hist edu latinoam. 2016;18(27):35-66. https://doi.org/10.19053/01227238.5508

40. Rodríguez AF, Páez RE, Altamirano EJ, Paguay FW, Rodríguez JC, Calero S. Nuevas perspectivas educativas orientadas a la promoción de la salud. Educ Med Super. 2017;31(4):1-11.

41. Aliaga E. Políticas de Promoción de la salud en el Perú: retos y perspectivas. Reporte final. Perú: Foro Salud y el Consorcio de Investigación Económica y Social [Internet]. 2003 [citado 2020 Marzo 29]. Recuperado a partir de http://www.consorcio.org/ Observatorio/publicaciones/aliaga.pdf

42. Giraldo A, Toro MY, Macías AM, Valencia CA, Palacio S. La Promoción de la Salud como Estrategia para el Fomento de Estilos de Vida Saludables. Hacia promoc Salud. 2010;15(1):128-43.

43. Premik M, Pavlekovic G, Kragelj LZ, Donev D. Healthy Public Policy. En: Donev D, Pavlekovic G, Kragelj LZ. A handbook for teachers, researchers, health professionals and decision makers. 1a ed. Alemania. Hans Jacobs Publishing Company; 2007. p. 38-57.

44. Ania Palacio, J. M. Guía para el diseño y la mejora de proyectos pedagógicos de educación y promoción de la salud. Madrid [Internet]. 2007 [citado 2020 Marzo 29]. Recuperado a partir de https:// www.mscbs.gob.es/profesionales/saludPublica/ prevPromocion/promocion/saludJovenes/docs/ proyectosPedagogicos 2007.pdf

45. Quintero EJ, de la Mella SF, Gómez L. La promoción de la salud y su vínculo con la prevención primaria. Medicent Electrón. 2017;21(2):101-111.

46. Anang P, Naujaat Elder EH, Gordon E, Gottlieb N, Bronson M. Building on strengths in Naujaat: the process of engaging Inuit youth in suicide prevention. Int J Circumpolar Health. 2019;78(2):1508321. https://doi.org/10.1080/22423982.2018.1508321

47. Ornelas IJ, Deschenie D, Jim J, Bishop S, Lombard $\mathrm{K}$, Beresford SA. Yéego Gardening! A Community Garden Intervention to Promote Health on the Navajo Nation. Prog Community Health Partnersh. 2017;11(4):417-425. https://doi.org/10.1353/ cpr.2017.0049

48. Nu J, Bersamin A. Collaborating With Alaska Native Communities to Design a Cultural Food Intervention to Address Nutrition Transition. Prog Community Health Partnersh. 2017;11(1):71-80. https://doi. org/10.1353/cpr.2017.0009

49. Harlow AF, Bohanna I, Clough A. A systematic review of evaluated suicide prevention programs targeting indigenous youth. Crisis. 2014;35(5):310-21. https:// doi.org/10.1027/0227-5910/a000265

50. Araujo M, Moraga C, Chpman E, Barreto J, Illanes E. Intervenciones para mejorar el acceso a los servicios de salud de los pueblos indígenas en las Américas. Rev Panam Salud Pública. 2016;40(5):371-381.

51. Rand JR. Inuit women's stories of strength: informing Inuit community-based HIV and STI prevention and sexual health promotion programming. Int $\mathbf{J}$ Circumpolar Health. 2016;75:32135. https://doi. org/10.3402/ijch.v75.32135

52. Leston J, Crisp C, Lee C, Rink E. An interview project with native American people: a community-based study to identify actionable steps to reduce health disparities. Public Health. 2019;176:82-91. https:// doi.org/10.1016/j.puhe.2018.12.002

53. Behm R. Determinantes económicos y sociales de la mortalidad en América Latina. Rev cub salud pública.2017;43(2):287-312.

54. paho.org [Internet].Colombia: Organización Panamericana de la Salud; 2016 [actualizado 30 mar 2020; citado 30 mar 2020]. Recuperado a partir de https://www.paho.org/hq/index.php?option=com_co ntent\&view $=$ article\&id $=12822$ : determinantessociales-salud-materna\&catid $=4717 \&$ Itemid $=39620$ \&lang $=$ es

55. Jiang L, Johnson A, Pratte K, Beals J, Bullock A, Manson SM; Special Diabetes Program for Indians Diabetes Prevention Program. Long-term Outcomes of Lifestyle Intervention to Prevent Diabetes in American Indian and Alaska Native Communities: The Special Diabetes Program for Indians Diabetes Prevention Program. Diabetes Care. 2018;41(7):14621470. https://doi.org/10.2337/dc17-2685

56. Crump CE, Letourneau RJ, Billie H, Zhang X, West B. Motor vehicle injury prevention in eight American Indian/Alaska Native communities: results from the 2010-2014 Centers for Disease Control and 
Prevention Tribal Motor Vehicle Injury Prevention Program. Public Health. 2019;176:29-35. https://doi. org/10.1016/j.puhe.2019.07.014

57. Gibson O, Lisy K, Davy C, Aromataris E, Kite E, Lockwood C, et al. Enablers and barriers to the implementation of primary health care interventions for Indigenous people with chronic diseases: a systematic review. Implement Sci. 2015;10(1):71. https://doi.org/10.1186/s13012-015-0261-x

58. Bersamin A, Izumi BT, Nu J, O'brien DM, Paschall M. Strengthening adolescents' connection to their traditional food system improves diet quality in remote Alaska Native communities: results from the Neqa Elicarvigmun Pilot Study. Transl Behav Med. 2019 Oct 1;9(5):952-961. https://doi.org/10.1093/ tbm/ibz087

59. Campero L, Atienzo E, Suarez L, Hernandez B, Villalobos A. Salud sexual y reproductiva de los adolescentes en México: evidencias y propuestas. Gac Méd Méx.2013;149(3):299-307.

60. Sanjuan-Meza XS, Padrón-Salas A, Valle-Luna P, Martínez-Granada S, Ortega-Velázquez A, CossíoTorres P. Reproductive health education program for Mexican women. Eur J Contracept Reprod Health Care. 2019;24(5):373-379. https://doi.org/10.1080/13 $\underline{625187.2019 .1656187}$

61. paho.org [Internet].Colombia: Organización Panamericana de la Salud; 2015 [Actualizado 30 mar 2020; citado 30 mar 2020]. Recuperado a partir de https:/www. paho.org/col/index.php?option $=$ com content\&v iew $=$ article $\& \mathrm{id}=2817$ :estrategia-de-cooperacioninteragencial-en-salud-materna-y-neonatal-indigena$\underline{2015-2019 \& \text { Itemid }=562}$ 